\title{
ОБЩИЕ КОНЦЕПЦИИ И ИДЕИ ТЕОРИИ ЭКСПЛУАТАЦИИ В КОНТЕКСТЕ ВЫЧИСЛИТЕЛЬНОЙ ТЕХНИКИ
}

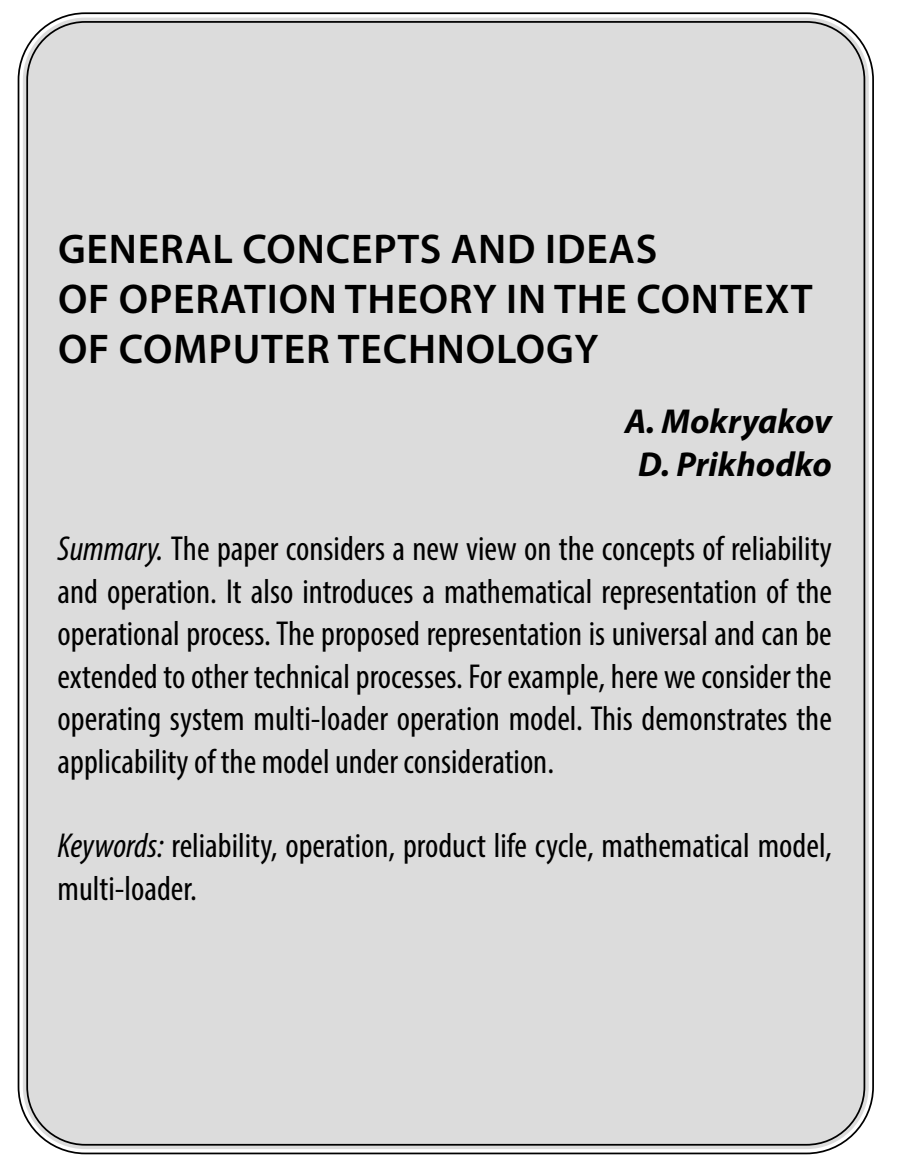

\section{Введение}

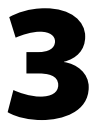

а неполные 80 лет электронные вычислительные системы прошли путь от научно-исследовательских инструментов до современной портативной техники, при этом непрерывно повышая качество её обслуживания, разнообразие исполняемых функций и удобство использования.

При этом произошла взаимная интеграция технических устройств, вследствие которой очень сильно изменилось общество. Например, сегодня уже практически все вещи интегрированы с интернетом: часы, телевизор, телефоны, умные очки и другие гаджеты. Это в свою очередь повышает требования к надёжности сложных электронных устройств, так как от одного могут зависеть многие устройства [1].

Перед тем, как начать непосредственное рассмотрение авторской точки зрения на надёжность устройств
Мокряков Алексей Викторович

К.ф.-м.н., дочент, Московский авиационный институт (национальный исследовательский университет); Российский государственный университет имени А. Н. Косыгина MokryakovAIVik@gmail.com

Приходько Дмитрий Игоревич Российский государственный университет имени

А. Н. Косыгина mitry1205@mail.ru

Аннотация. В работе рассматривается новый взгляд на понятия надёжности и эксплуатации. Также здесь вводится математическое представление 06 эксплуатационном процессе. Предложенное представление универсальное и может быть распространено на другие технические процессы. Например, здесь рассматривается модель эксплуатации мультизагрузчика операционных систем. Что демонстрирует применимость рассматриваемой модели.

Ключевые слова: надёжность, эксплуатация, жизненный цикл изделия, математическая модель, мультизагрузчик.

и их эксплуатацию, необходимо произвести разбор тех теорий, которые были предложены ранее.

\section{Краткий исторический экскурс}

В общем виде имеет смысл рассматривать понятие эксплуатация, в непосредственной связи с показателями работы системы. Одним из самых популярных, является понятие «надежность». И в таком случае, уместно рассмотреть эволюцию «надёжности» в целом.

В кратком варианте историю понятия надежности можно рассмотреть таким образом:

В 1935 г. инженер Я.М. Туровер рассмотрел термин «надёжность» в отношении электротехнических систем. Он также отметил важность представления «надежности» не как качественного, а количественного параметра, для оценки, которого стоит использовать методы теории вероятностей. 
В середине 50-60-х годов были выдвинуты основные варианты определения «надежность», заложившие основу современному определению надёжности, различные версии этого термина во временной динамике выглядят так [2]:

1. Надежность (reliability) - вероятность выполнения устройством своих функций с учетом своих требований в течение заданного интервала времени.

2. Надежность - свойство объекта, заключающееся в выполнении им определенных задач в конкретных условиях эксплуатации (объект - предмет целевого назначения, вещь, рассматриваемая на всех этапах жизненного цикла).

3. Надёжность - интегральная функция распределения вероятности безотказной работы с начала работы до первого отказа.

4. Надежность - способность элемента (системы) обеспечивать и сохранять в условиях эксплуатации заданные значения обобщенных координат и передаточного коэффициента в течение определенного отрезка времени в пределах установленных допусков.

5. Надежность - среднее время работы данного образца между двумя неисправностями.

6. Надежность - это вероятность безотказной работы.

В 1975 г. ГОСТ 13377-75 (Надежность в технике. Термины и определения) установил следующее определение надёжности: свойство объекта выполнять заданные функции, сохраняя во времени значения установленных эксплуатационных показателей в заданных пределах, соответствующих заданным режимам и условиям использования, технического обслуживания, ремонтов, хранения и транспортирования.

В 1983 г. формулировку этого понятия изменили [3]: свойство объекта сохранять во времени в установленных пределах значения всех основных параметров, характеризующих способность выполнять требуемые функции в определённых режимах и условиях применения, технического обслуживания, починки, хранения и транспортирования.

В 1992 г. ИСО 188402 обозначил надежность, как способность изделия выполнять требуемые функции в заданных условиях в течение заданного периода времени.

В начале 21 века ощущается недостаток внимания к понятию «надежность», а также проявилась тенденция к исключению этого понятия из стандартов менеджмента качества [4].
Теперь же уместно будет дать определение эксплуатации: это стадия жизненного цикла изделия, на которой реализуется, поддерживается или восстанавливается его качество.

Однако в рамках вышеизложенного обзора источников возникает вопрос: как производить оценку эксплуатации в рамках сильно меняющейся окружающей среды, в которых система должна работать?

Данный вопрос связан с тем, что параметры окружающей среды в таком случае меняются значительно. Например, бортовой компьютер, который одинаково должен функционировать в широком рабочем диапазоне: начиная с сверхнизких температур в космосе и радиации, и заканчивая работой внутри космического корабля. Другой пример: компьютерная установка, которая работает на атомной электростанции и управляет выработкой энергии.

Надежность в рассматриваемом варианте - это гарантированная работа системы во всех требуемых условиях окружающей среды, с применением необходимых для этого механизмов адаптации, требуемой временной продолжительности, и обработкой большинства «прогнозируемых» отказов.

А эксплуатация соответственно - этап жизненного цикла работы системы, на котором гарантировано выполнение с требуемой точностью вычислений и надёжностью выполнения требуемых задач при помощи внутренних возможностей системы, существование адаптивных внешних механизмов для защиты от воздействия меняющейся окружающей среды, а также механизмов ремонта и аварийной обработки большинства ошибок работы системы.

В дальнейшем мы будем рассматривать надёжность и эксплуатацию систем в рамках зависимости от 2-х механизмов: внешней среды и адаптивных механизмов.

\section{Рассмотрение \\ механизмов эксплуатации}

Для начала стоит отметить, что вопрос эксплуатации сам по себе является довольно интересным.

Например, каким образом возможно определить, где можно использовать компьютер, а где этого делать категорически запрещено, поскольку идут в учёт многие дополнительные факторы, которые необходимо учитывать заранее.

При этом следует отметить, что некоторые внешние факторы можно нивелировать с целью повышения ка- 
чества выполнения требуемых от систем задач. Это достигается за счет применения специальных адаптивных механизмов, которые являются в большинстве случаев типовыми, но иногда требуются специальные дополнительные механизмы (например, водонепроницаемость или ударопрочность телефонов).

Ну и наконец необходимо ответить на самый важный вопрос: какие характеристики нужны для того, чтобы получить желаемый результат с учётом требований к системе, при этом сохраняя самые важные свойства, которые присущи данной вычислительной системе? Этот вопрос является ключевым.

Ответ на этот вопрос можно начать с того, чтобы разделить непрерывный процесс эксплуатации на ряд последовательных процессов, используемых в своих зонах ответственности.

Таким образом в упрощённом варианте мы можно расписать весь процесс на три простых составляющих, которые и будут характеризовать весь эксплуатационный процесс.

Процесс в данном случае подразумевается, как некоторое уравнение, которое характеризует взаимодействие системы с окружающим миром, который условно представляется как некоторый набор параметров.

Следовательно, задачу эксплуатации системы можно представить в виде следующих процессов:

1. Процесс, описывающий начальные параметры системы и связь при начальных условиях. Это перечень факторов окружающей среды, которые существуют вне зависимости от наличия адаптивных механизмов, в которых функционирует система. Примеры: радиация, влажность погоды и другие.

2. Процесс, описывающий итоговые условия окружающей среды с учётом применения адаптивных механизмов. Это всевозможные теплоотводы, корпус, и т.п. Пример: процессор в ПК выделяет очень много тепла при работе, и чтобы можно было работать на больших мощностях, которыми обладает современная вычислительная техникаиспользуется кулеры и пр. виды систем охлаждения. И таким образом, от того, как именно будет данная система, зависит возможность использования на конкретной мощности процессора.

3. Процесс самой работы системы. В данном процессе рассматривается работа системы в итоге получившихся условий среды, которые удалось скомпенсировать, и рассматриваются вопросы эксплуатации самой системы. Например: какая мощность процессора требуется для выполне- ния требуемой задачи. В этом процессе нет надобности в проведении анализа по факторам внешней среды, так как их учёт ведётся в предыдущем процессе.

$$
F(p(i))=\left\{\begin{array}{l}
\operatorname{In}(p(i)), \text { если } p(i)<V_{\text {in1 }}(i) \\
\operatorname{Wrk}(g(i)), \text { если } V_{\text {in }}(i)<g(i)<V_{\text {out } 1}(i) \\
\operatorname{Out}(r(i)), \text { если } r(i)>V_{\text {in1 }}(i)
\end{array}\right.
$$

В математическом виде это можно выразить таким образом:

Здесь $\mathrm{p}(\mathrm{i}), \mathrm{g}(\mathrm{i}), \mathrm{r}(\mathrm{i})$ - цепной набор параметров, подаваемых на функцию, при этом Vin $1(p(i))$ и Vout1 (p(i)) вектора границ функции требуемой системы на заданных условиях.

Следует отметить, что наличие ограничений на вышеизложенные функции вытекают из того факта, что система существует в физическом мире, и поэтому на неё накладываются определенные ограничения, которые и отображены как расположенные по другую сторону от значений функций.

\section{Прелставление математической мохели}

Одним из видов представления рассматриваемой модели, может являться трёхмерный график, а если мы хотим учесть временную продолжительность, то возможно представление модели в виде четырёхмерного графика, получая таким образом требуемую динамику работы, и при этом четвертая координата является временной осью.

Однако, в некоторых случаях, например для упрощённого анализа, когда требуется спроектировать простейшие системы, можно обойтись и меньшим числом процессов. Например, когда проектируем систему автоматической установки мультизагрузчиков на носитель [5-6].

Учитывая функциональное наполнение современных ОС, нет нужды описывать, что сегодня почти все актуальные версии загрузчиков поддерживают большинство современных версий ОС (например семейство ОС Windows, начиная c Windows 7, и новее, и зачастую даже NT5X семейство) [7].

Следовательно, для построения модели для анализа работы 3-й процесс не существенен и не требуется его моделирование, на основании того, что третий процесс в указанной модели будет одинаковым для всех рассматриваемых версий мультизагрузчиков. 


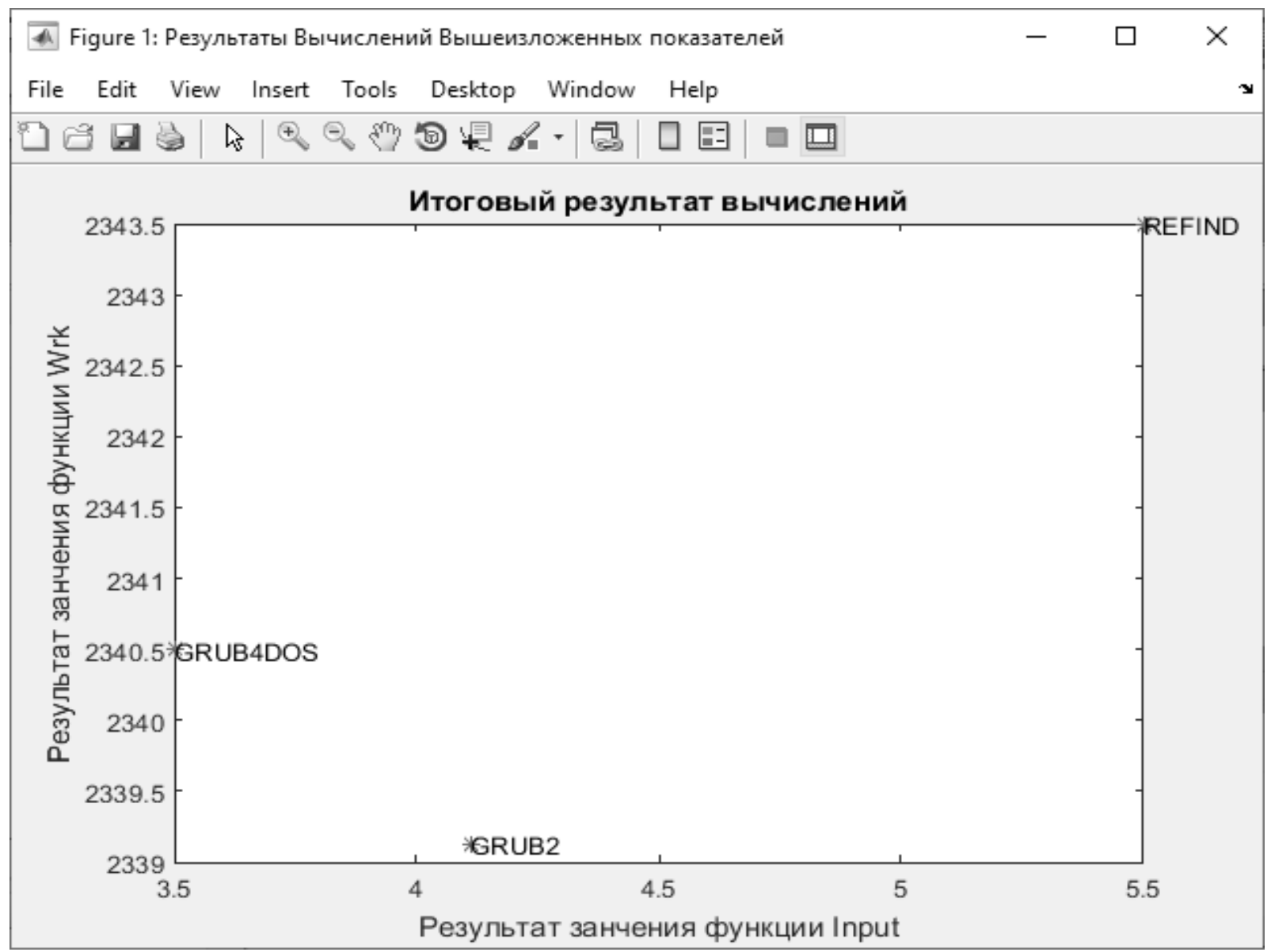

Рис. 1. Итоговый график результатов

С учётом данного упрощения, достаточно рассмотрения системы в рамках 2-х процессов, а следовательно, и представление модели возможно в виде графика на двухмерной системе координат. На рисунке 1 представлены результаты рассматриваемой модели.

Для реализации был использован математический пакет моделирования matlab, причём данная реализация демонстрирует указанные состояния процессов. По каждой координатной оси расположено значение того или итого процесса, который в данном случае является оценочным для соответствующих процессов.

\section{Эак^ючение}

Предложенная концепция, позволяет полностью отобразить эксплуатацию системы с учётом различных факторов окружающей среды. На её основе возможно разработать модель защиты различных вычислительных систем, для которых необходимо повышения пределов эксплуатации в сложных условиях. Также рассматриваемая модель эксплуатации позволяет создать модель процессов с необходимыми эксплуатационными характеристиками, которые требуются заказчику.

\section{ЛИТЕРАТУРА}

1. Курносов М.Г., Пазников А. А. Основы теории функционирования распределенных вычислительных систем. Новосибирск: Автограф, 2015.52 с.

2. Тимошенков С.П., Симонов Б.М., Горошко В.Н. Основы теории надежности: учебник и практикум для академического бакалавриата. М: Юрайт, 2019. 445 C.

3. ГОСТ 27.002-89. Надёжность техники. М.: Издательство стандартов, 1989.

4. Чебоксаров А. Н. Основы теории надежности и диагностика: курс лекций. Омск: СибАДИ, 2012.76 с.

5. Приходько Д. И. Математические модели упрощения загрузки // Инновационное развитие современной науки: проблемы, закономерности, перспективы. Сборник статей VI Международной научно-практической конференции: в 2 ч. 2018. С. 17-23. 
6. Приходько Д. И. Методы оптимизации мультизагрузочных и установочных носителей // Научная дискуссия современной молодёжи: актуальные вопросы, достижения и инновации. Сборник статей III Международной научно-практической конференции: в 2 ч. 2018. С. 13-19. Режим доступа: naukaip.ru/ wp-content/uploads/2018/03/MK-303-Сборник-Часть-1.pdf.

7. Приходько Д. И. Прикладные модели загрузчиков операционных систем // Прорывные научные исследования: проблемы, закономерности, перспективы. Сборник статей Х Международной научно-практической конференции. 2018. С. 31-37. Режим доступа: http://naukaip.ru/wp-content/uploads/2018/03/ Сборник-MK-306.pdf.

○ Мокряков Алексей Викторович ( MokryakovAIVik@gmail.com ), Приходько Дмитрий Игоревич ( mitry1205@mail.ru).

Журнал «Современная наука: актуальные проблемы теории и практики»
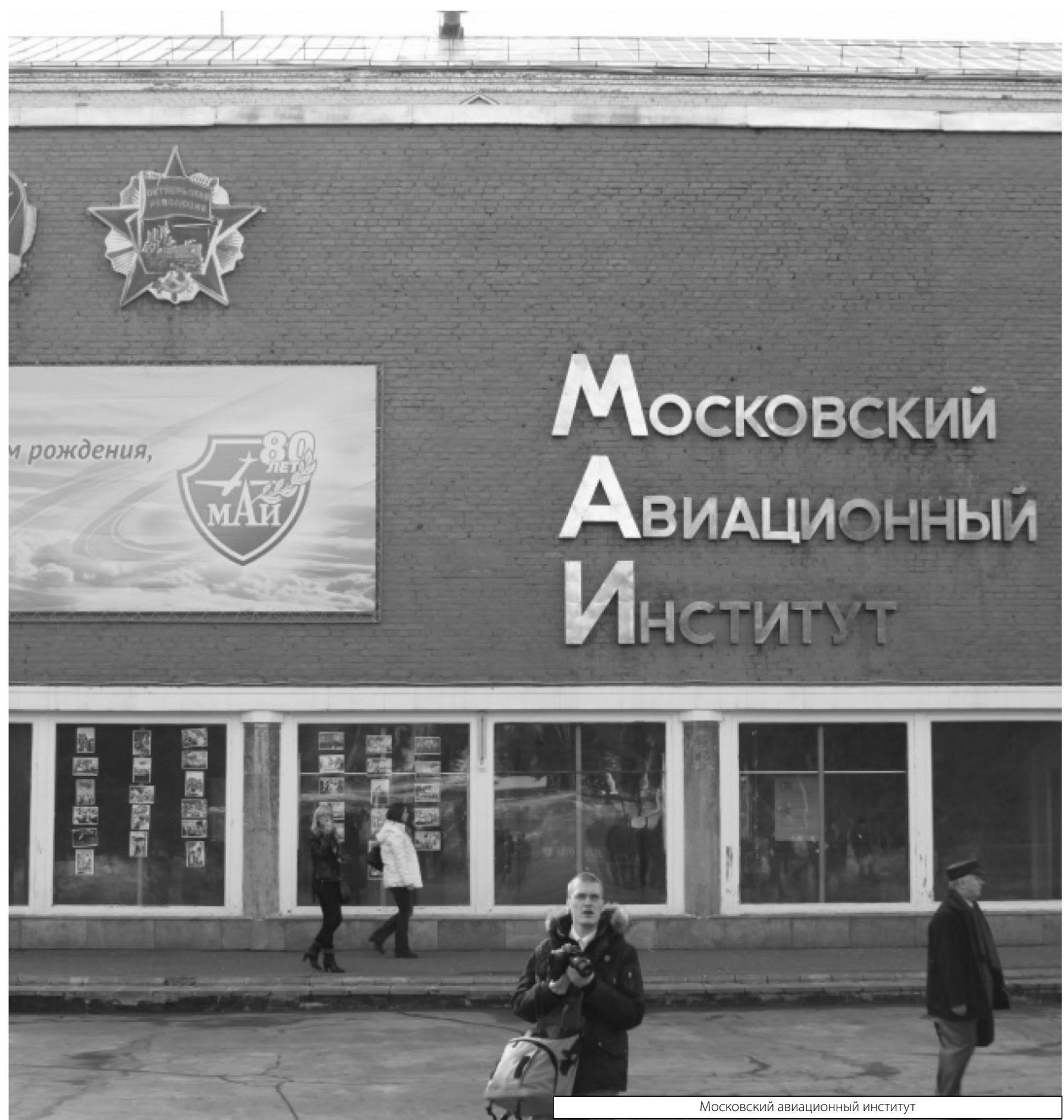